\title{
Identifying the heterogeneity of COPD by V/P SPECT: a new tool for improving the diagnosis of parenchymal defects and grading the severity of small airways disease
}

\author{
This article was published in the following Dove Press journal: \\ International Journal of COPD \\ 26 May 2017 \\ Number of times this article has been viewed
}

M Bajc'
Y Chen
JWang
XY Li $^{4}$
WM Shen
CZ Wang
H Huang
A Lindqvist
XY He

'Department of Clinical Science Physiology and Nuclear Medicine, Skåne University Hospital, Lund, Sweden; ${ }^{2}$ Respiratory Department, Changzheng Hospital, Shanghai, ${ }^{3}$ Respiratory Department, Xinqiao Hospital, Chongqing, ${ }^{4}$ Respiratory Department, Huadong Hospital, Shanghai, China; ${ }^{5}$ Department of Pulmonary Medicine, Heart and Lung Center, Helsinki University Hospital and Helsinki University, Helsinki, Finland; ${ }^{6}$ Suzhou University Affiliated Tumor Hospital, Wuxi, China
Correspondence: M Bajc Department of Clinical Physiology and Nuclear Medicine, Department of Imaging and Physiology, Skåne University Hospital and Lund University, Getingevägen 4, S-22185 Lund, Sweden

Tel +46 46 I 73303

Email marika.bajc@med.lu.se
Introduction: Airway obstruction and possible concomitant pulmonary diseases in COPD cannot be identified conventionally with any single diagnostic tool. We aimed to diagnose and grade COPD severity and identify pulmonary comorbidities associated with COPD with ventilation/perfusion single-photon emission computed tomography (V/P SPECT) using Technegas as the functional ventilation imaging agent.

Methods: 94 COPD patients (aged 43-86 years, Global Initiative for Chronic Obstructive Lung Disease (GOLD) stages I-IV) were examined with V/P SPECT and spirometry. Ventilation and perfusion defects were analyzed blindly according to the European guidelines. Penetration grade of Technegas in V SPECT measured the degree of obstructive small airways disease. Total preserved lung function and penetration grade of Technegas in V SPECT were assessed by V/P SPECT and compared to GOLD stages and spirometry.

Results: Signs of small airway obstruction in the ventilation SPECT images were found in 92 patients. Emphysema was identified in 81 patients. Two patients had no signs of COPD, but both of them had a pulmonary embolism, and in one of them we also suspected a lung tumor. The penetration grade of Technegas in V SPECT and total preserved lung function correlated significantly to GOLD stages ( $r=0.63$ and -0.60 , respectively, $P<0.0001$ ). V/P SPECT identified pulmonary embolism in 30 patients $(32 \%)$. A pattern typical for heart failure was present in 26 patients (28\%). Parenchymal changes typical for pneumonia or lung tumor were present in several cases.

Conclusion: V/P SPECT, using Technegas as the functional ventilation imaging agent, is a new tool to diagnose COPD and to grade its severity. Additionally, it revealed heterogeneity of COPD caused by pulmonary comorbidities. The characteristics of these comorbidities suggest their significant impact in clarifying symptoms, and also their influence on the prognosis.

Keywords: V/P SPECT, COPD, imaging interpretation criteria, Technegas, pulmonary comorbidities

\section{Introduction}

COPD is a major cause of morbidity and mortality. The global number of COPD cases was estimated to be 384 million in 2010, with a prevalence of $11.7 \%$. $^{1}$ In China, COPD is the second leading cause of morbidity and mortality. In 2004, the overall prevalence of COPD was $8.2 \%$ in seven Chinese provinces and cities. ${ }^{2}$ Both smoking and biomass fuel consumption are important etiological factors. ${ }^{3,4}$ Programs for improved patient management in clinical practice have been organized in China. ${ }^{5}$ 
COPD is characterized by airflow limitation and abnormal inflammatory response. ${ }^{6,7}$ The changes include increased airway resistance due to airway obstruction, inflammation and luminal plugs, and increased compliance caused by parenchymal destruction and loss of alveolar attachments. There are also vascular changes due to inflammatory remodeling, with progressive vascular occlusion and loss of vasculature in areas with emphysematous destruction. Comorbidities like left heart failure (LHF), lung cancer, pulmonary embolism (PE), and atherosclerosis are common. ${ }^{8,9}$ Hence, COPD is a heterogeneous condition, and the clinical presentation, pathophysiology, disease progression, and response to therapy vary between patients. ${ }^{10}$

In general, COPD is diagnosed and graded by spirometer indices like $\mathrm{FEV}_{1}$ (forced expiratory volume in 1 second), $\mathrm{FVC}$ (forced vital capacity), and $\mathrm{FEV}_{1}$ in relation to $\mathrm{FVC}$, or FEV\%. ${ }^{7}$ Lowering of FEV\% measures the degree of airflow obstruction, predominantly in large and intermediate airways, but provides no explanation of the underlying pathophysiology. ${ }^{11}$ It is therefore generally accepted that $\mathrm{FEV}_{1}$ by itself cannot describe the complexity of COPD and that $\mathrm{FEV}_{1}$ alone cannot be used for optimal assessment and management of the disease. . $^{72}$

Individualized treatment of COPD is growing, and there is a need for better ways to categorize patients into different phenotypes, and in the best case endotypes, to be able to optimize therapy, follow and predict disease progression, and measure response to therapy. ${ }^{10}$ Different imaging modalities are generating interest within the field of COPD. ${ }^{13}$

Tomographic lung scintigraphy (ventilation/perfusion single-photon emission computed tomography: V/P SPECT) is a nuclear medicine investigation technique that gives a 3-dimensional functional map of the ventilation and perfusion of the lungs. Its primary use is in the diagnosis and follow-up of PE. ${ }^{14-16}$ The introduction of ultrafine aerosols like Technegas has expanded the field of application for V/P SPECT, and airway obstruction is no longer considered a problem for PE diagnosis, which was the case for previous hydrosoluble aerosols. ${ }^{17}$ Moreover, V/P SPECT has been shown to have applications in COPD and to be more sensitive than CT and $\mathrm{FEV}_{1}$ in detecting early airway changes. ${ }^{18-20} \mathrm{~V} / \mathrm{P}$ SPECT can be used to differentiate between healthy controls and COPD patients and to grade the degree of small airway obstruction in COPD. ${ }^{19,21}$ Furthermore, it may be used to semi-quantitatively assess the degree of lung function reduction in obstructive lung disease and PE. ${ }^{19,22}$

We aimed to evaluate V/P SPECT in the diagnosis of COPD, in grading the severity of COPD, and in specifying the role of additional pulmonary comorbidities inducing heterogeneity.

\section{Patients and methods}

Ninety four patients with a clinical diagnosis of COPD, based on the 2011 revision of Global Initiative for Chronic Obstructive Lung Disease (GOLD) criteria with a postbronchodilator ratio of $\mathrm{FEV}_{1}$ over $\mathrm{FVC}<0.70$, were enrolled in 3 hospitals in Shanghai and Chongqing in an international multicentre trial among patients who had a current or former smoking history ( $>10$ pack years) or a verified biomass exposure, who were aged 40 years or older, and who had stable disease and no exacerbation or respiratory infection within 6 weeks prior to study entry. The patients had no allergic diathesis or a history of bronchial asthma, domiciliary oxygen, and no history of pulmonary resection, or concomitant major illness, or difficulties in cooperation. Pregnant or breast-feeding women were excluded.

All patients signed an informed consent form for participation prior to study entry, according to the ICH Harmonized Tripartite Guideline for Good Clinical Practice. This prospective study was approved by the Human Research Ethics Committee of Changzheng Hospital and Huadong Hospital in Shanghai (the Shanghai Changzheng Hospital biomedical science research ethics committee) and Xinqiao Hospital in Chongqing (No 2 affiliate hospital of the 3rd Military Medical University medical ethics committee).

Clinical symptoms, diagnoses, and medications were listed in the case record form. The subjects were reevaluated at the study visit by post-bronchodilator spirometry, performed according to ATS/ERS standards. ${ }^{23}$ A MasterScope ${ }^{\text {TM }}$ spirometer (Jaeger, Würzburg, Germany) was used to measure pulmonary function by $\mathrm{FEV}_{1}, \mathrm{FVC}$, and their ratio $\left(\mathrm{FEV}_{1} /\right.$ $\mathrm{FVC}=\mathrm{FEV} \%$ ). GOLD criteria were used at the study visit to grade the severity of airflow limitation in COPD patients to clinical stage I or mild COPD ( $\mathrm{FEV}_{1}>80 \%$ predicted), stage II or moderate COPD $\left(\mathrm{FEV}_{1} 50 \%-80 \%\right.$ predicted), stage III or severe COPD $\left(\mathrm{FEV}_{1} 30 \%-50 \%\right.$ predicted), and stage IV or very severe COPD $\left(\mathrm{FEV}_{1}<30 \%\right.$ predicted). ${ }^{24}$

\section{V/P SPECT protocol}

$\mathrm{V} / \mathrm{P}$ SPECT imaging was performed in accordance with the recommendations of the European Association of Nuclear Medicine. ${ }^{25,26}$ In short, a large-field-of-view dual-head gamma-camera with a low-energy, all-purpose collimator was used. Technegas (Cyclomedica Ltd, Kingsgrove, NSW, Australia) was inhaled until $30 \mathrm{MBq}$ had reached the lungs, 
and ventilation tomography followed. After that, with the patient in a carefully maintained supine position, $140 \mathrm{MBq}$ 99mTc-MAA was slowly injected intravenously. Then, perfusion tomography was performed. Acquisition was performed in a $64 \times 64$ matrix, zoomed to a pixel size of $6.8 \mathrm{~mm}$ with 128 projections over 360 degrees. For the ventilation study, each step was of 10 s duration, and for the perfusion study, $5 \mathrm{~s}$ duration were used for each step. Reconstruction was performed using ordered subsets expectation maximization with 8 subsets and 2 iterations.

$\mathrm{V} / \mathrm{P}$ SPECT was interpreted centrally by experienced nuclear medicine physicians, blinded to other information, and read according to the guidelines of the EANM. ${ }^{25}$ The degree of obstructive small airways disease was visually graded according to a predefined 4-point scale (0-3) based on the ventilatory impairment shown by the penetration grade of Technegas in V SPECT (Vgrade), as described earlier. ${ }^{27}$ $\mathrm{V} / \mathrm{P}$ SPECT images were reviewed according to a clinical routine assessing the presence of PE, LHF, emphysema, pneumonia, or suspicion of tumor. ${ }^{15,25,28,29}$

Ventilation/perfusion defects were quantified by counting segments or subsegments showing complete or reduced ventilation and/or perfusion defects and were expressed in $\%$ of the total lung parenchyma. ${ }^{19,22,30}$ The degree of obstructive disease, if present, was described as mild (approximately affecting $20 \%$ of the lung function), moderate $(20 \%-50 \%)$, or severe $(>50 \%)$. The extent of matched, mismatched, and reverse mismatched defects was expressed as a percentage of the total lung volume. The sum of these was used to estimate the extent of the total reduction in lung function. To be regarded as an area with fully preserved lung function, both ventilation and perfusion had to be normal and matched. Total preserved lung function was quantified semiquantitatively and described in $\%$ of the total estimated lung volume (TPLF\%).

\section{Statistics}

Prism 6 software (GraphPad Software, Inc. La Jolla, CA, USA) was used for statistical analysis. Baseline characterization of the patients and the groups of patients formed on the basis of classification of their disease were assessed by descriptive analyses in terms of age, gender, medical history, etc. Correlation analyses were done using Pearson correlation coefficients $(r)$. Linear regression analysis was used to evaluate the relationship between the V/P SPECT measures and pulmonary function tests. For group comparisons, analysis of variance followed by post-tests or Mann-Whitney test for comparison of two groups were applied. Results are expressed as mean and standard deviation or mean and $95 \%$ confidence intervals. A value of $P$-value less than 0.05 was considered to be significant.

\section{Results}

Patient characteristics are shown in Table 1. Eighty-eight percent of the patients were smokers (28\% currently smoking), and $12 \%$ were exposed to biomass smoke only. Eighty nine percent of the patients were males. Seventy seven percent of the patients were treated with a combination therapy of inhaled corticosteroid and long-acting beta-agonist (ICS+LABA), 50\% were treated with a long-acting muscarinic antagonist (LAMA), and $13 \%$ used theophylline. LABA was never given as a monotherapy. Seventeen percent of the patients used LAMA as a monotherapy, 6\% used theophylline combined with SABA and/or SAMA. Short-acting beta-agonists (SABAs) as needed and/or short-acting muscarinic antagonists (SAMAs) as needed were used as rescue therapy. With this medication, the post-bronchodilatation $\mathrm{FEV}_{1}$ and $\mathrm{FVC}$ of the patients ranged from 21\%-108\% (mean 50\%) and 28\%-125\% (mean 82\%), respectively, of the predicted value. FEV\% ranged between 25\%-86\% (mean 51\%). Typical clinical symptoms were cough (91\%), wheeze $(89 \%)$, expectoration $(77 \%)$, chest tightness (45\%), and breathlessness (31\%). Fever (5\%), chest pain (1\%), or hemoptysis (1\%) were occasionally present. GOLD grading of COPD severity was mild in $6 \%$, moderate in $35 \%$, severe in $49 \%$, and very severe in $10 \%$ of cases. Fifty-seven percent of patients had experienced at least one exacerbation of COPD during the past year. The number of exacerbations per patient within the past 12 months ranged from 0-6 (mean 1).

Abnormal Vgrade as a sign of obstructive small airways disease in the ventilation SPECT images was found in 92 patients (98\%, Figure 1). This ventilatory impairment

Table I Characteristics of the patients

\begin{tabular}{lll}
\hline 94 COPD patients & Mean \pm SD & Range \\
\hline Age (years) & $68 \pm 9$ & $43-86$ \\
Height $(\mathrm{cm})$ & $164 \pm 7$ & $146-178$ \\
Weight $(\mathrm{kg})$ & $66 \pm 11$ & $38-98$ \\
BMI $\left(\mathrm{kg} / \mathrm{m}^{2}\right)$ & $24 \pm 4$ & $16-35$ \\
Pack years & $33 \pm 21$ & $0-104$ \\
Number of exacerbations within the & $1.1 \pm 1.3$ & $0-6$ \\
past I2 months & & \\
Spirometry & & \\
- Post-bronchodilatation $\mathrm{FEV}$, & $50 \% \pm 18 \%$ pred & $21 \%-108 \%$ pred \\
- Post-bronchodilatation $\mathrm{FVC}$ & $82 \% \pm 22 \%$ pred & $28 \%-125 \%$ pred \\
- Post-bronchodilatation $\mathrm{FEV} / \mathrm{FVC}$ & $51 \% \pm 15 \%$ & $25 \%-86 \%$ \\
- Acute bronchodilatation response & $6.3 \% \pm 7.8 \%$ & $-9 \%-43 \%$ \\
\hline
\end{tabular}

Abbreviations: BMI, body mass index; FEV ${ }_{1}$, forced expiratory volume in I second; FVC, forced vital capacity; SD, standard deviation. 

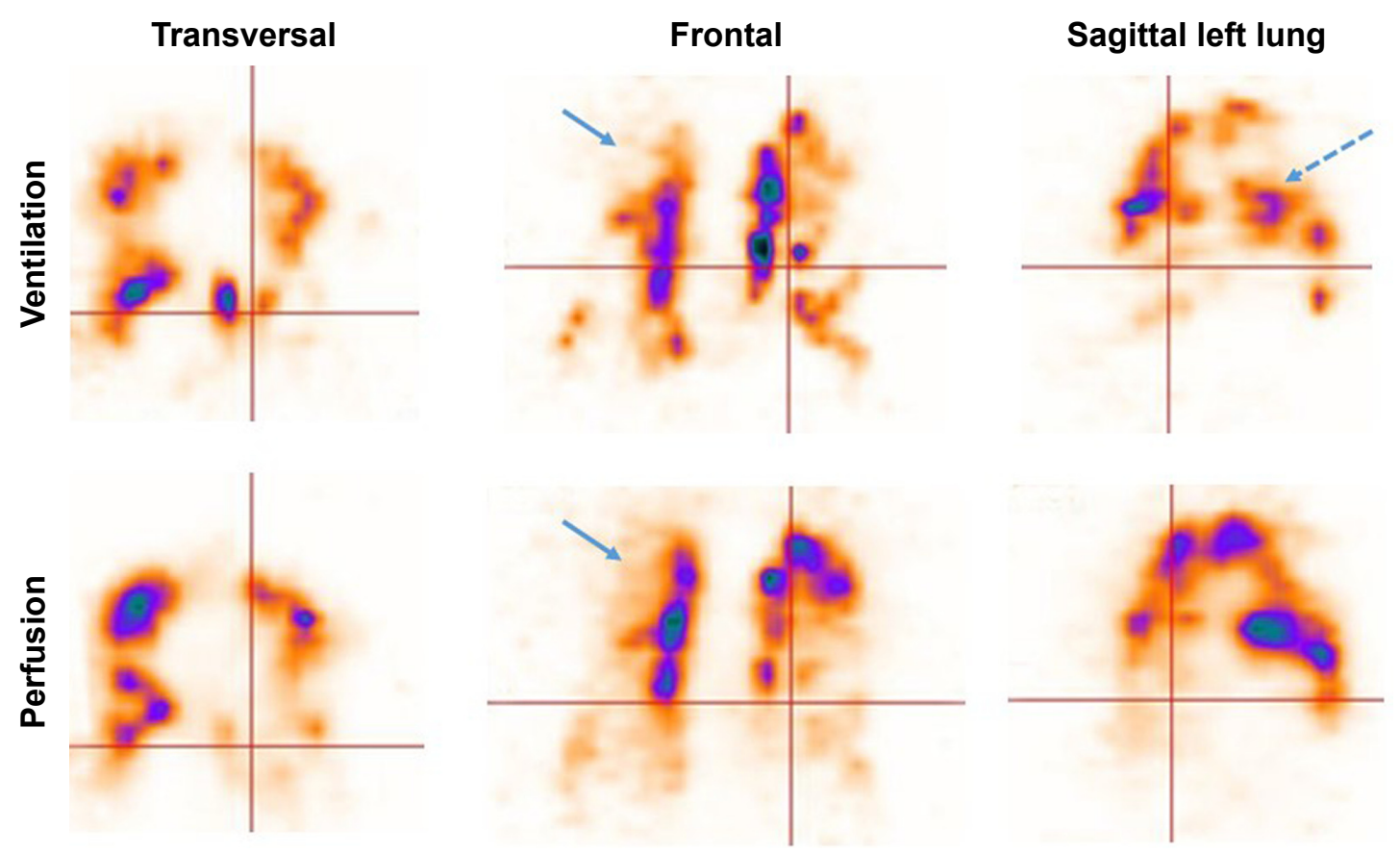

Figure I V/P SPECT of a patient with a severe COPD (GOLD 3).

Notes: On the frontal slice, uneven distribution of ventilation and absent ventilation are seen, as is perfusion in the upper lobe (blue arrows indicating emphysema). Hot spots represent changes in small airway disease (dotted blue arrow on sagittal slice).

Abbreviations: GOLD, Global Initiative for Chronic Obstructive Lung Disease; V/P SPECT, ventilation/perfusion single-photon emission computed tomography.

was graded mild $(3 \%)$, moderate $(40 \%)$, or severe $(55 \%)$. Two patients $(2 \%)$ included in the study had no signs of COPD in the V/P SPECT. However, we found that both of these patients had a PE, and in one of them we suspected a lung tumor. Vgrade and TPLF\% agreed well with the GOLD stages ( $r=0.63$ and -0.60 , respectively, $P<0.0001$, Figure 2 ). Vgrade and TPLF\% showed a weaker correlation with the pulmonary function tests (Figure 3). The V/P SPECT analysis tended to diagnose ventilation defects earlier than spirometry. Vgrade 3 had significantly lower pulmonary function values in spirometry than Vgrade 2 (Figure 4). Emphysema was identified in 81 patients $(86 \%)$. TPLF\% varied from $15 \%-80 \%$ (mean $38 \%$ ).

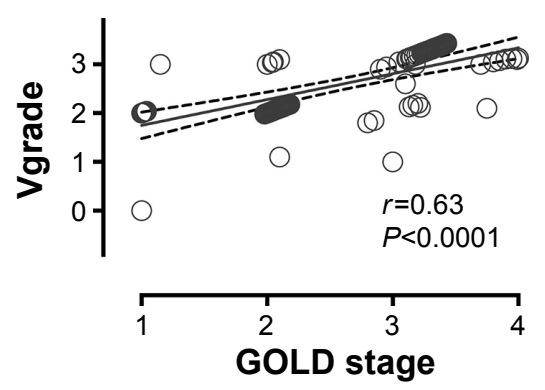

PE was found in 30 patients (32\%), and LHF was present in 26 patients (28\%). Findings suspicious for tumors were identified in 15 patients $(16 \%)$, and pneumonia was reported in 4 patients (4\%). Several patients had more than one comorbidity (Figure 5).

In the ordinary clinical examination, $23 \%$ of the patients were found to have emphysema, 4\% PE, 6\% lung cancer, $5 \%$ pneumonia, $1 \%$ acute bronchitis, $1 \%$ pulmonary tuberculosis under treatment, and $1 \%$ bronchiectasis. Their concomitant extrapulmonary diagnoses included hypertension (19\%), coronary artery disease (9\%), and chronic heart failure (2\%). Other diagnoses include diabetes, rheumatoid arthritis, and gastrointestinal diseases (altogether 19\%).

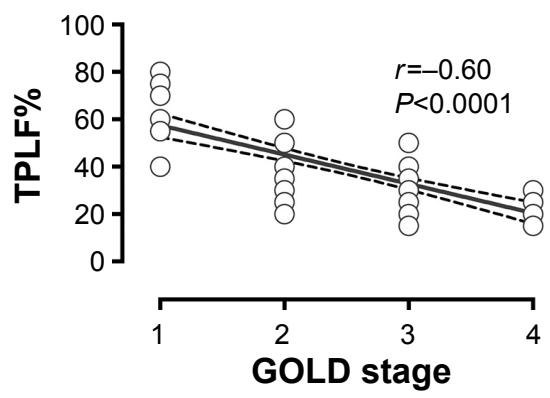

Figure 2 Correlation between Vgrade and TPLF\% and GOLD stages.

Note: Pearson correlation coefficients and linear regression lines with best-fit values (continuous line) and $95 \%$ confidence intervals (dotted lines) are presented. Abbreviations: GOLD, Global Initiative for Chronic Obstructive Lung Disease; TPLF\%, total preserved lung function; SPECT, single-photon emission computed tomography; Vgrade, penetration grade of Technegas in V SPECT. 

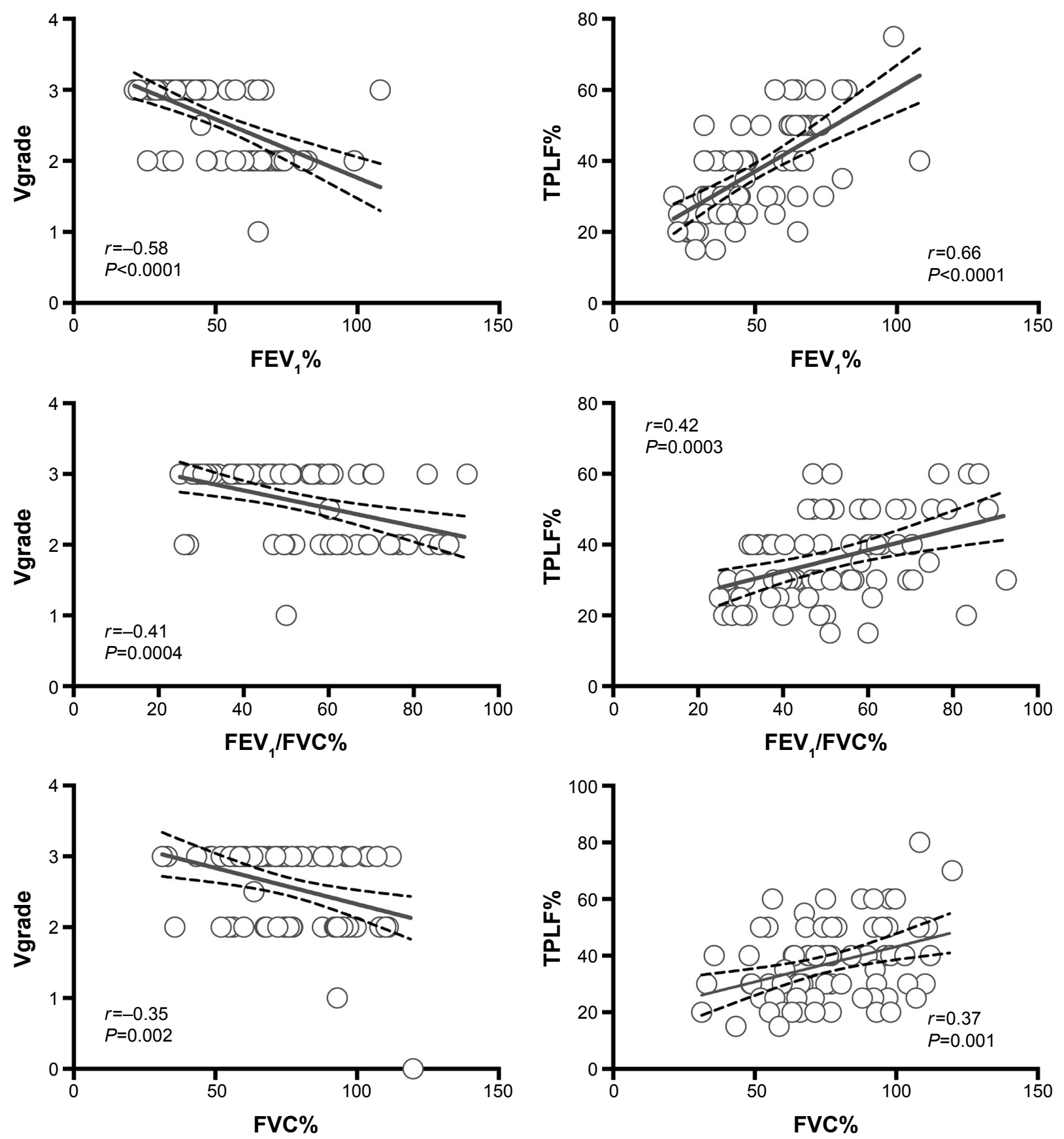

Figure 3 Correlation between Vgrade and TPLF\% and results of the technically accepted and reproducible pulmonary lung function tests.

Note: Pearson's correlation coefficients and linear regression lines with best-fit values (continuous line) and $95 \%$ confidence intervals (dotted lines) are presented.

Abbreviations: $\mathrm{FEV}_{1}$, forced expiratory volume in I second; FVC, forced vital capacity; TPLF\%, total preserved lung function; SPECT, single-photon emission computed tomography; Vgrade, penetration grade of Technegas in V SPECT.

\section{Discussion}

$\mathrm{V} / \mathrm{P}$ SPECT is a new tool to diagnose COPD and to grade its severity. ${ }^{27}$ In this study, Vgrade and TPLF\% agreed well with GOLD stages. ${ }^{19,27} \mathrm{~V} / \mathrm{P}$ SPECT also revealed pulmonary comorbidities with vascular and ventilatory defects, which contribute to the heterogeneity of COPD. Emphysema was a more frequent finding than clinically expected. All V/P SPECT findings were diagnostic.
The small hydrophobic aerosol property of Technegas enables its penetrations into the smallest conducting airways, and then finally by diffusion reaching the alveolar level. ${ }^{31}$ Technegas penetration depends on the small airway function, and bronchial deposition increases in small airway disease allowing detection of airway obstruction earlier by Technegas ventilation image than by spirometry. ${ }^{19,32}$ In a study by Jobse et al, ${ }^{18}$ small airways disease was predicted 

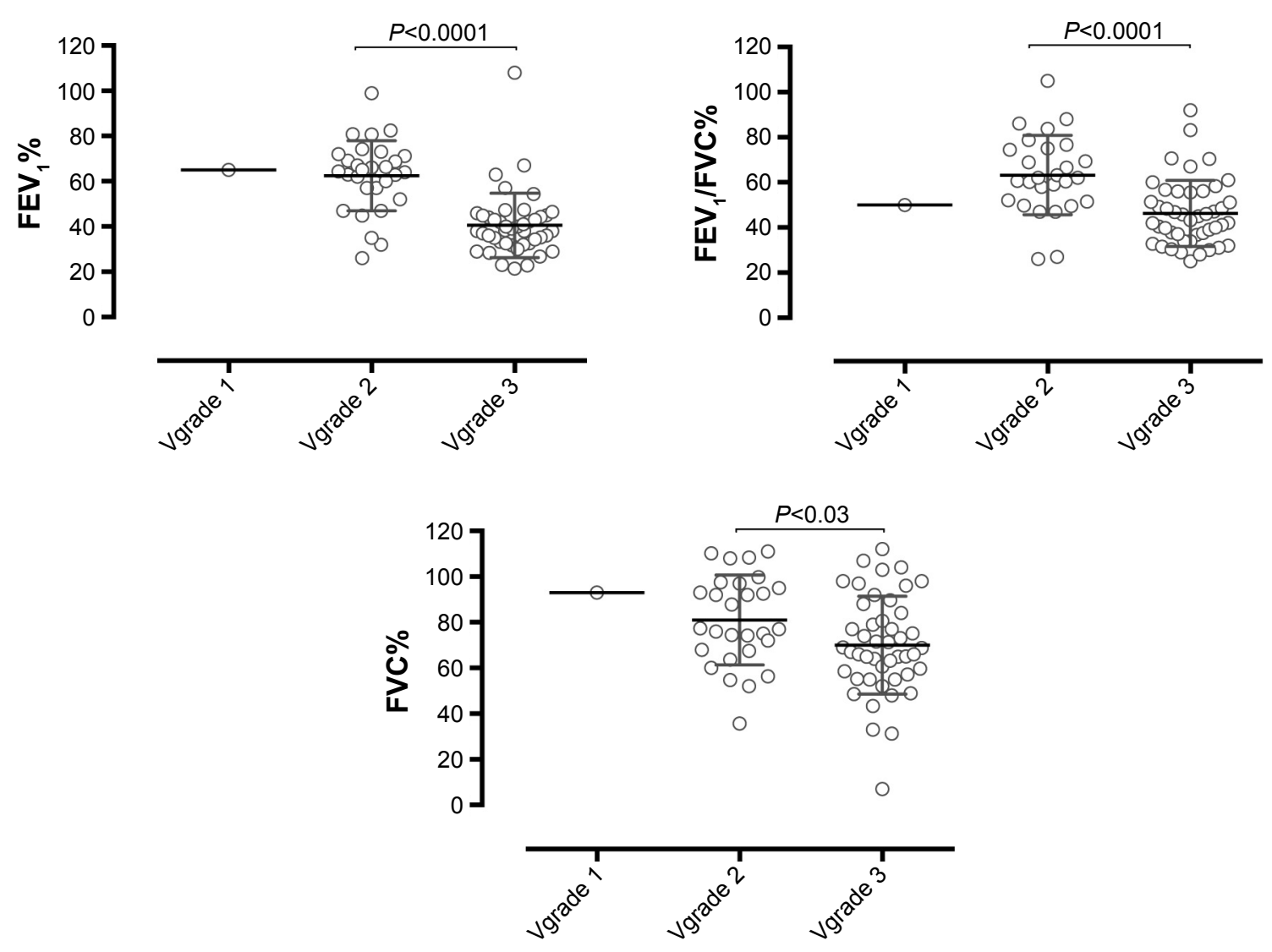

Figure 4 Vgrade I, 2, and 3 in relation to the technically accepted and reproducible lung function test results ( $n=75)$.

Note: The plots of measurements with the mean and the $95 \%$ confidence intervals are shown.

Abbreviations: $\mathrm{FEV}_{1}$, forced expiratory volume in I second; FVC, forced vital capacity; SPECT, single-photon emission computed tomography; Vgrade, penetration grade of Technegas in V SPECT.

by the ventilation/perfusion pattern on V/P SPECT. The authors suggest that the hot spots representing exaggerated deposition of Technegas in the airways appear when narrowing is sufficiently severe to cause airflow limitations (Figure 1). Our results regarding Vgrade and its relation to different degrees of obstructive small airways disease agree with earlier published data. ${ }^{11,19,27,32-34}$ In the present study, most of the patients had an advanced obstructive disease, favoring the good correlation between the V/P SPECT and spirometry in measuring airway obstruction.

PE was the most frequent comorbidity and was seen in $32 \%$ of patients. The perfusion defects were typically segmental or subsegmental mismatches, and some were probably residuals after earlier acute untreated PEs. In two cases, the V/P SPECT detected vascular defects (and also one suspicion of a lung tumor) in the absence of any signs of COPD on V/P SPECT. Both patients were included into the study on the basis of symptoms and obstructive lung function findings suggesting COPD. The vascular defect might explain the patients' dyspneic symptoms in these cases.
One patient actually received a clinical diagnosis of PE and lung cancer. The PE was a previously unknown finding in another patient. Asthma with reversible obstruction cannot be excluded in these cases.

An acute PE may often remain undiagnosed in COPD patients. Usually, PE is diagnosed at the time of COPD exacerbation with no clear etiology. ${ }^{35}$ In a recent systematic review by Aleva et al, ${ }^{36}$ the pooled prevalence of PE was $16 \%$ (confidence interval [CI] 8.3\%-25.8\%) in a total of 880 COPD patients. PE seems to increase mortality and length of hospital admission. ${ }^{36}$

In our study, only four patients were known to have had PE before V/P SPECT imaging, and three of them still showed perfusion defects in this study. Earlier studies show that even treated patients might have residual perfusion defects. ${ }^{16,37,38}$ It is known that the vascular response is often impaired in COPD patients. ${ }^{39}$

The second most important comorbidity was signs of LHF, present in $28 \%$ of the COPD patients. Generally, it is known that COPD and chronic heart failure frequently coexist. ${ }^{40} \mathrm{In}$ 


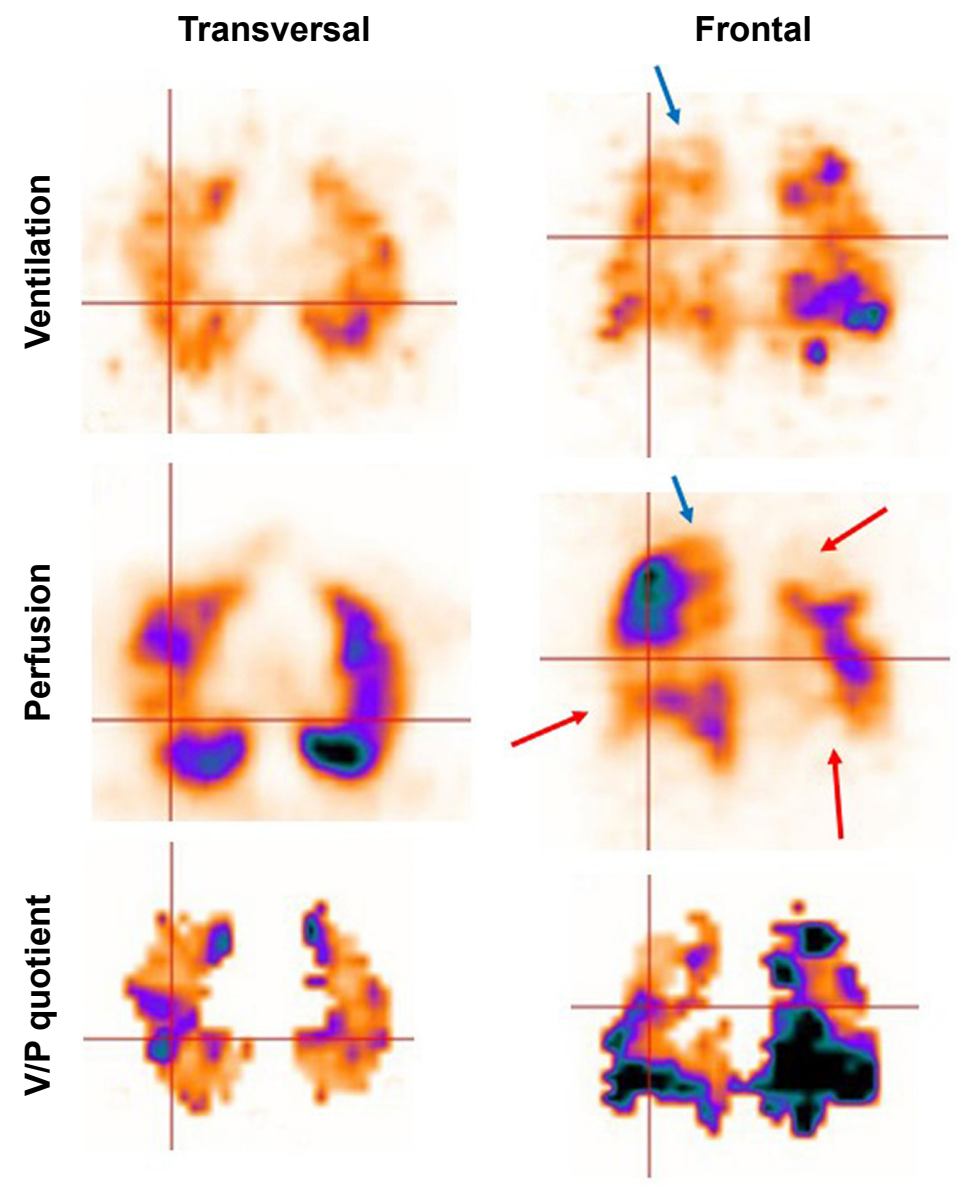

\section{Sagittal right lung}
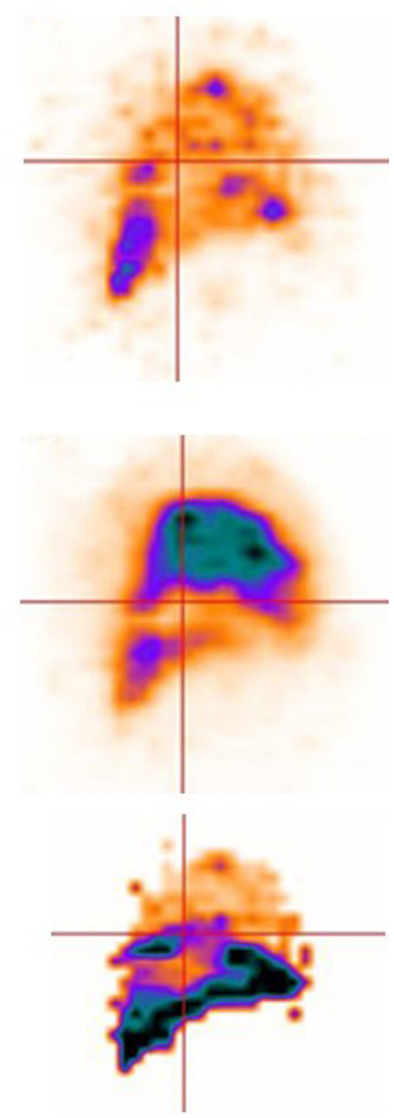

Figure 5 V/P SPECT of a patient with a severe COPD (GOLD 3).

Note: Uneven distribution of ventilation with hot spots (indicating obstructive small airways disease), area with absent ventilation and perfusion in the upper lobe (blue arrows indicating emphysema), as well as area with absent perfusion and preserved ventilation (mismatch - red arrows indicating pulmonary embolism).

Abbreviations: GOLD, Global Initiative for Chronic Obstructive Lung Disease; V/P SPECT, ventilation/perfusion single-photon emission computed tomography.

patients hospitalized with heart failure, COPD was diagnosed in $30 \%-36 \%$ of the patients. ${ }^{41,42}$ Obviously, this clinical condition is a diagnostic challenge owing to the fact that patients with COPD, LHF, and PE have similar and nonspecific symptoms in the form of dyspnea, cough, exercise intolerance, and fatigue. Chest X-ray, electrocardiogram, CT, and spirometry might be misleading diagnostic procedures for this complex status. LHF is known to have a prognostic impact. ${ }^{43,44}$

These results highlight the heterogeneity of COPD. As many as $58(62 \%)$ subjects among the total number of 94 studied COPD patients had 1 or more comorbidities that were capable of being identified with V/P SPECT. These findings might have an impact on treatment in this subgroup of COPD patients. In some patients, the reason for exacerbations might be an acute PE or an acute LHF. We do not know of any method, other than V/P SPECT, that might diagnose both the severity and heterogeneity of COPD with pulmonary comorbidities. Therefore, the study raises the question of how frequently the V/P SPECT procedure is indicated for the diagnosis and classification of COPD or COPD exacerbation.

The limitation of this study is the dominance of men among the enrolled patients and the absence of a control group. High-resolution CT could be of added value for diagnosing nodular changes and to allow better delineation of emphysema. Further studies are required to measure the degree of obstructive small airways disease by V/P SPECT, particularly in "healthy smokers" with a normal pulmonary function on spirometry.

\section{Conclusion}

V/P SPECT could diagnose and grade severity of COPD, and estimate preserved lung function. Even more important, V/P SPECT appears to be a unique physiological method to reveal pulmonary comorbidities with vascular and ventilatory defects, which contribute to the heterogeneity of COPD. 
The characteristics of these comorbidities suggest their impact on the symptoms, treatment, and prognosis of patients.

\section{Acknowledgments}

The authors express their gratitude to Mr Michael Guo for the excellent management of the communication between and data collection from the study centers. The authors appreciate the dedication of the patients and the personnel and physicians at the Departments of Pulmonology as well as at the Departments of Nuclear Medicine of the study centers to the study protocol and procedures. This study was financially supported by Region of Scania (ALF) and Scania University foundation Skåne University Hospital (SUS Fonder). MB has, during the last 3 years, received research grants from Cyclomedica.

\section{Author contributions}

MB and XY He designed the study. Doctors Y Chen, J Wang, XY Li, WM Shen, CZ Wang, and H Huang carried out the study, contributed to data acquisition and analysis, and provided administrative support. MB was a central reader. AL contributed to the study design, statistical analysis, and manuscript drafting. All authors read and approved the final version of the manuscript. All authors contributed toward data analysis, drafting and critically revising the paper and agree to be accountable for all aspects of the work.

\section{Disclosure}

The authors report no conflicts of interest in this work.

\section{References}

1. Adeloye D, Chua S, Lee C, et al. Global and regional estimates of COPD prevalence: systematic review and meta-analysis. J Glob Health. 2015;5(2):020415.

2. Zhong N, Wang C, Yao W, et al. Prevalence of chronic obstructive pulmonary disease in China: a large, population-based survey. Am J Respir Crit Care Med. 2007;176(8):753-760.

3. Zhou Y, Wang C, Yao W, et al. COPD in Chinese nonsmokers. Eur Respir J. 2009;33(3):509-518.

4. Liu S, Zhou Y, Wang X, et al. Biomass fuels are the probable risk factor for chronic obstructive pulmonary disease in rural South China. Thorax. 2007;62(10):889-897.

5. Guan WJ, Ran PX, Zhong NS. Prevention and management of COPD in China: successes and major challenges. Lancet Respir Med. 2016; 4(6):428-430.

6. Celli BR, MacNee W, Force AET. Standards for the diagnosis and treatment of patients with COPD: a summary of the ATS/ERS position paper. Eur Respir J. 2004;23(6):932-946.

7. Vestbo J, Hurd SS, Agusti AG, et al. Global strategy for the diagnosis, management, and prevention of chronic obstructive pulmonary disease: GOLD executive summary. Am J Respir Crit Care Med. 2013; 187(4):347-365.

8. Lofdahl CG. COPD and co-morbidities, with special emphasis on cardiovascular conditions. Clin Respir J. 2008;2(Suppl 1):59-63.

9. Wedzicha JA, Hurst JR. Chronic obstructive pulmonary disease exacerbation and risk of pulmonary embolism. Thorax. 2007;62(2):103-104.
10. Han MK, Agusti A, Calverley PM, et al. Chronic obstructive pulmonary disease phenotypes: the future of COPD. Am J Respir Crit Care Med. 2010;182(5):598-604.

11. Hogg JC, Chu F, Utokaparch S, et al. The nature of small-airway obstruction in chronic obstructive pulmonary disease. $N$ Engl J Med. 2004; 350(26):2645-2653.

12. Jarenback L, Ankerst J, Bjermer L, Tufvesson E. Flow-volume parameters in COPD related to extended measurements of lung volume, diffusion, and resistance. Pulm Med. 2013;2013:782052.

13. Schuster DP. The opportunities and challenges of developing imaging biomarkers to study lung function and disease. Am J Respir Crit Care Med. 2007;176(3):224-230.

14. Bajc M, Neilly JB, Miniati M, Schuemichen C, Meignan M, Jonson B. EANM guidelines for ventilation/perfusion scintigraphy: Part 2. Algorithms and clinical considerations for diagnosis of pulmonary emboli with V/P(SPECT) and MDCT. Eur J Nucl Med Mol Imaging. 2009; 36(9):1528-1538.

15. Bajc M, Olsson B, Palmer J, Jonson B. Ventilation/Perfusion SPECT for diagnostics of pulmonary embolism in clinical practice. $J$ Intern Med. 2008;264(4):379-387.

16. Begic A, Jogi J, Hadziredzepovic A, Kucukalic-Selimovic E, BegovicHadzimuratovic S, Bajc M. Tomographic ventilation/perfusion lung scintigraphy in the monitoring of the effect of treatment in pulmonary embolism: serial follow-up over a 6-month period. Nucl Med Commun. 2011;32(6):508-514.

17. Jogi J, Jonson B, Ekberg M, Bajc M. Ventilation-perfusion SPECT with 99mTc-DTPA versus technegas: a head-to-head study in obstructive and nonobstructive disease. J Nucl Med. 2010;51(5):735-741.

18. Jobse BN, Rhem RG, McCurry CA, Wang IQ, Labiris NR. Imaging lung function in mice using SPECT/CT and per-voxel analysis. PLoS One. 2012;7(8):e42187.

19. Jogi J, Ekberg M, Jonson B, Bozovic G, Bajc M. Ventilation/perfusion SPECT in chronic obstructive pulmonary disease: an evaluation by reference to symptoms, spirometric lung function and emphysema, as assessed with HRCT. Eur J Nucl Med Mol Imaging. 2011;93: 1344-1352.

20. Satoh K, Nakano S, Tanabe M, et al. A clinical comparison between Technegas SPECT, CT, and pulmonary function tests in patients with emphysema. Radiat Med. 1997;15(5):277-282.

21. Norberg P, Persson HL, Carlsson GA, et al. Quantitative lung SPECT applied on simulated early COPD and humans with advanced COPD. EJNMMI Res. 2013;3(1):28.

22. Olsson CG, Bitzen U, Olsson B, et al. Outpatient tinzaparin therapy in pulmonary embolism quantified with ventilation/perfusion scintigraphy. Med Sci Monit. 2006;12(2):PI9-PI13.

23. Miller MR, Hankinson J, Brusasco V, et al. Standardisation of spirometry. Eur Respir J. 2005;26(2):319-338.

24. Pauwels RA, Buist AS, Ma P, Jenkins CR, Hurd SS, Committee GS. Global strategy for the diagnosis, management, and prevention of chronic obstructive pulmonary disease: National Heart, Lung, and Blood Institute and World Health Organization Global Initiative for Chronic Obstructive Lung Disease (GOLD): executive summary. Respir Care. 2001;46(8):798-825.

25. Bajc M, Neilly JB, Miniati M, Schuemichen C, Meignan M, Jonson B. EANM guidelines for ventilation/perfusion scintigraphy: Part 1. Pulmonary imaging with ventilation/perfusion single photon emission tomography. Eur J Nucl Med Mol Imaging. 2009;36(8): $1356-1370$.

26. Palmer J, Bitzen U, Jonson B, Bajc M. Comprehensive ventilation/ perfusion SPECT. J Nucl Med. 2001;42(8):1288-1294.

27. Bajc M, Markstad H, Jarenback L, Tufvesson E, Bjermer L, Jogi J. Grading obstructive lung disease using tomographic pulmonary scintigraphy in patients with chronic obstructive pulmonary disease (COPD) and long-term smokers. Ann Nucl Med. 2015;29(1):91-99.

28. Begic A, Opankovic E, Cukic V, Lindqvist A, Miniati M, Bajc M. Ancillary findings assessed by ventilation/perfusion tomography. Impact and clinical outcome in patients with suspected pulmonary embolism. Nuklearmedizin. 2015;54(5):223-230. 
29. Jogi J, Palmer J, Jonson B, Bajc M. Heart failure diagnostics based on ventilation/perfusion single photon emission computed tomography pattern and quantitative perfusion gradients. Nucl Med Commun. 2008; 29(8):666-673.

30. Elf JE, Jogi J, Bajc M. Home treatment of patients with small to medium sized acute pulmonary embolism. J Thromb Thrombolysis. 2015; 39(2)166-172.

31. Bajc M, Neilly B, Miniati M, Mortensen J, Jonson B. Methodology for ventilation/perfusion SPECT. Semin Nucl Med. 2010;40(6): 415-425.

32. Norberg P, Persson HL, Schmekel B, et al. Does quantitative lung SPECT detect lung abnormalities earlier than lung function tests? Results of a pilot study. EJNMMI Res. 2014;4(1):39.

33. Pellegrino R, Biggi A, Papaleo A, Camuzzini G, Rodarte JR, Brusasco V. Regional expiratory flow limitation studied with Technegas in asthma. J Appl Physiol (1985). 2001;91(5):2190-2198.

34. Jobse BN, Rhem RG, Wang IQ, Counter WB, Stampfli MR, Labiris NR. Detection of lung dysfunction using ventilation and perfusion SPECT in a mouse model of chronic cigarette smoke exposure. J Nucl Med. 2013;54(4):616-623.

35. Tillie-Leblond I, Marquette CH, Perez T, et al. Pulmonary embolism in patients with unexplained exacerbation of chronic obstructive pulmonary disease: prevalence and risk factors. Ann Intern Med. 2006; 144(6):390-396.

36. Aleva FE, Voets LW, Simons SO, de Mast Q, van der Ven AJ, Heijdra YF. Prevalence and localization of pulmonary embolism in unexplained acute exacerbations of COPD: a systematic review and meta-analysis. Chest. 2017;151(3):544-554.
37. Alhadad A, Miniati M, Alhadad H, Gottsater A, Bajc M. The value of tomographic ventilation/perfusion scintigraphy (V/PSPECT) for follow-up and prediction of recurrence in pulmonary embolism. Thromb Res. 2012;130(6):877-881.

38. Wartski M, Collignon MA. Incomplete recovery of lung perfusion after 3 months in patients with acute pulmonary embolism treated with antithrombotic agents. THESEE Study Group. Tinzaparin ou Heparin Standard: Evaluation dans 1'Embolie Pulmonaire Study. J Nucl Med. 2000;41(6):1043-1048.

39. Lami D, Cellai AP, Antonucci E, et al. Residual perfusion defects in patients with pulmonary embolism are related to impaired fibrinolytic capacity. Thromb Res. 2014;134(3):737-741.

40. Hawkins NM, Virani S, Ceconi C. Heart failure and chronic obstructive pulmonary disease: the challenges facing physicians and health services. Eur Heart J. 2013;34(36):2795-2803.

41. Boschetto P, Fucili A, Stendardo M, et al. Occurrence and impact of chronic obstructive pulmonary disease in elderly patients with stable heart failure. Respirology. 2013;18(1):125-130.

42. Iversen KK, Kjaergaard J, Akkan D, et al. Chronic obstructive pulmonary disease in patients admitted with heart failure. J Intern Med. 2008;264(4):361-369.

43. Arnaudis B, Lairez O, Escamilla R, et al. Impact of chronic obstructive pulmonary disease severity on symptoms and prognosis in patients with systolic heart failure. Clin Res Cardiol. 2012;101(9):717-726.

44. Mentz RJ, Schmidt PH, Kwasny MJ, et al. The impact of chronic obstructive pulmonary disease in patients hospitalized for worsening heart failure with reduced ejection fraction: an analysis of the EVEREST Trial. J Card Fail. 2012;18(7):515-523.
International Journal of COPD

\section{Publish your work in this journal}

The International Journal of COPD is an international, peer-reviewed journal of therapeutics and pharmacology focusing on concise rapid reporting of clinical studies and reviews in COPD. Special focus is given to the pathophysiological processes underlying the disease, intervention programs, patient focused education, and self management protocols.

\section{Dovepress}

This journal is indexed on PubMed Central, MedLine and CAS. The manuscript management system is completely online and includes a very quick and fair peer-review system, which is all easy to use. Visit http://www.dovepress.com/testimonials.php to read real quotes from published authors. 